\title{
Aus dem Zentralvorstand
}

\section{An seiner Sitzung Anfang Mai hat der Zentralvorstand der FMH ...}

... entschieden, praktische Empfehlungen zum Swissmedic-Leitfaden über die gute Praxis in der Aufbereitung von sterilen Medizinprodukten zu erarbeiten.

... sich über das Spitzengespräch mit santésuisse informieren lassen:

- Mittlerweile ist die BIP separat bis Ende Jahr geregelt.

- Man hat sich über das Vorgehen bei der Verbesserung des Tarifwesens geeinigt, welches auch ein neues Kapitel Grundversorgung enthalten wird.

... sich einverstanden erklärt mit den vorgeschlagenen Anpassungen des Reglements «Schweizerische
Kommission für Berufsentwicklung und Qualität» hinsichtlich der MPA-Ausbildung.

... beschlossen, die Autorenrechte des Praxislehrgangs für die Ausbildung von MPA zu übernehmen und EMH mit Druck und Vertrieb zu beauftragen.

... der Neuformulierung der Lohnempfehlung im Mustervertrag für Praxisstellvertretungen zugestimmt. Sie lautet: «Wir empfehlen, sich bei der Festlegung des Salärs an den Löhnen von Ärzten der entsprechenden Erfahrungsstufe zu orientieren.»

... die Nachfrage nach Weiterbildungstiteln zur Kenntnis genommen, die höher ausgefallen ist als erwartet.

\section{Délibérations du Comité central}

\section{Lors de sa séance début mai, le Comité central de la FMH a...}

... décidé d'élaborer des recommandations pratiques au sujet de la réglementation sur la préparation des dispositifs médicaux stériles.

... reçu des informations sur la discussion au sommet avec santésuisse:

- l'indemnité de dérangement en cas de visite est maintenant réglée pour elle-même jusqu'à la fin de l'année.

- les parties se sont entendues sur la marche à suivre pour optimiser la structure tarifaire qui comprendra un nouveau chapitre consacré à la médecine générale.

... accepté les propositions de modification du règlement de la «Commission suisse pour le dévelop- pement professionnel et la qualité» en vue de la formation des assistantes médicales.

... décidé de prendre à sa charge les droits d'auteur du cours de formation des assistantes médicales et d'en attribuer la publication et la diffusion aux éditions EMH.

... approuvé la reformulation des recommandations de salaire dans la convention cadre sur les remplacements au cabinet médical: «Pour fixer le salaire, nous recommandons de se référer aux salaires des médecins bénéficiant du même niveau d'expérience.»

... pris note de l'augmentation plus élevée que prévu du nombre de demandes de titres. 